The nexus between socio-cultural norms and safe sexual choices such as condom use places young migrants' at-risk cohort with receiving countries, as they are currently outside of the realms of socio-cultural contexts, with higher education expectations and delay of marriage customs.

Conclusion There is a need for targeted and redesign of condom promotion programmes taking into account the current realities of transitioning young migrants who lives outside of the boundaries of current socio-cultural paradigms.

\section{P2 STAFF EXPERIENCES OF WORKING WITH CHILDREN AND YOUNG PEOPLE (CYP) EXPERIENCING PERSISTENT UNEXPLAINED PHYSICAL SYMPTOMS (PUPS) IN THE CONTEXT OF A NEW BIOPSYCHOSOCIAL MODEL OF CARE}

L Holmyard*, A Boyd. Psychology, Exeter University, Exeter, UK

\subsection{6/bmjpo-2019-RCPCH-SAHM.10}

Background An estimated 10-25\% of Children and Young People (CYP) experience PUPS (Hinton \& Kirk, 2016). PUPS is a complex phenomenon comprising an interplay of biopsychosocial factors. Healthcare professionals working in 'physical' health settings often struggle to find a helpful way forward with this group of CYP (Furness et al., 2009). This can lead to break-down in communication between families and professionals and increase medicalisation of symptoms (Furness et al., 2009).

Aims This study aimed to explore healthcare professional's experiences of caring for CYP presenting with PUPS following the implementation of a specialist biopsychosocial MDT.

Method Individual, semi-structured interviews were conducted with 7 health professionals who had referred CYP into the MDT. An inductive approach to thematic analysis (TA) (Joffe \& Yardley, 2004) was used to identify salient themes from the interviews.

Findings Two overarching themes emerged from the interviews, each of which contained numerous sub-themes.Firstly, interviewees drew attention to care for CYP presenting with PUPS being in a state of 'Disintegration'prior to the implementation of the MDT, both structurally and relationally. Interviewees went on to discuss how implementation of the MDT had allowed for greater structural and relational 'Integration'. Interviewees reflected on how increased structural and relational integration allowed space for mind-body integration for CYP.

Conclusions The positive experiences of professionals in this study, in addition to literature recommending a biopsychosocial approach to PPS (Lazarus, 2003) and supportive national policy (NHS England, 2016) create a strong case for a multi-disciplinary approach to caring for CYP presenting with PPS. This should include professionals with expertise in physical health, alongside those with psychological expertise and those concerned with the social welfare of CYP.

\section{REFERENCES}

1. Furness P, Glazebrook C, Tay J, Abbas K, Slaveska-Hollis K. Medically unexplained physical symptoms in children: exploring hospital staff perceptions. Clinical child psychology and psychiatry 2009;14(4):575-587.

2. Hinton D, Kirk S. Families' and healthcare professionals' perceptions of healthcare services for children and young people with medically unexplained symptoms: a narrative review of the literature. Health \& social care in the community 2016;24 (1):12-26.
3. Joffe H, Yardley L. Content and Thematic Analysis. In Research methods for Clinical Health Psychology 2004;55-56.London: Sage.

4. Lazarus AA. (2003). Multimodal Therapy: A Primer. Retrieved from http://www zurinstitute.com/multimodaltherapy.html

\section{P3 TWO WAY TEXT-MESSAGING IMPROVES HIV OUTCOMES FOR HIV-POSITIVE YOUTH WITH GAPS IN CARE}

${ }^{1} \mathrm{D}$ Gerke, ${ }^{2} \mathrm{~J}$ Glotfelty, ${ }^{2} \mathrm{~J}$ Schlueter, ${ }^{2} \mathrm{M}$ Freshman, ${ }^{2} \mathrm{~S}$ Slovacek, ${ }^{3} \mathrm{M}$ Jonson-Reid, ${ }^{2} \mathrm{~K}$ Plax* ${ }^{1}$ School of Social Work, University of Denver, Denver, USA; ${ }^{2}$ Pediatrics, Washington University School of Medicine, St Louis, USA; ${ }^{3}$ School of Social Work, Washington University, St Louis, USA

\subsection{6/bmjpo-2019-RCPCH-SAHM.11}

Aims Youth are overrepresented in the number of new HIV cases and fare worse across the HIV care continuum. To address these challenges, we implemented a text-messaging intervention to increase engagement in care and improve viral suppression (VLS) rates. The aim of this study was to determine if intervention participation increased achieving or maintaining VLS after 6 months.

Methods We recruited $100 \mathrm{HIV}$-positive youth receiving care in our program and meeting at least one additional criterion (newly diagnosed; not linked to care; out of care more than six months in last 2 years; viral load $>200$ copies $/ \mathrm{mL}$ ). The automated two-way text-messaging intervention included medication reminders, appointment reminders, a housing/utilities needs assessment and mood checks. Participant challenges reported triggered action alerts to case managers who responded via text or phone. Results were examined over 6 months.

Results Participants in the intervention were mostly male (79\%), Black (91\%), and YMSM (71\%), with an average age of $24.58(\mathrm{SD}=2.88)$. Youth in our intervention group experienced trauma (29. 4\% reported intmate partner violence and $34.3 \%$ experienced sexual assault) and participated in survival sex $(22.5 \%$ traded sex and $28.4 \%$ received payment for sex). $46.2 \%$ of participants reported alcohol use and $55.8 \%$ reported marijuana use several times per month. $81 \%$ of youth participated in 89,120 text exchanges. Text responses triggered 395 alerts. The most common alerts were for missed medication, missed appointments and housing issues. Twentyfive participants newly achieved VLS and 35 maintained VLS during the 6 months. A significantly greater proportion of participants were virally suppressed at 6 months versus baseline $(p=0.018)$

Conclusion Even with high rates of adversity experiences this two-way texting intervention improved VLS for youth and for those with gaps in care.

\section{P4 USING PROVIDER TRAINING AND ELECTRONIC SCREENING AND FEEDBACK TO IMPROVE PREVENTIVE CARE FOR ADOLESCENTS IN PRIMARY CARE}

${ }^{1,2}$ CA McCarty*, 1,2 LP Richardson, 'E Parker, ${ }^{1} \mathrm{M}$ Stepanchak, ${ }^{2}$ J Stout. ${ }^{1}$ Center for Child Health, Behavior and Development, Seattle Children's Hospital, Seattle, USA; ${ }^{2}$ Pediatrics, University of Washington, Seattle, USA

\subsection{6/bmjpo-2019-RCPCH-SAHM.12}

Aims Emerging evidence suggests that the consistency of provider counseling can be increased through training and the use of screening tools; yet, we know little about the 